BULLETIN Bulletin hispanique

HISPANIQUE Université Michel de Montaigne Bordeaux

$115-2$ | 2013

Les traductions vieillissent-elles ?

\title{
Le livre de l'Intranquillité de Fernando Pessoa
}

traduit en français par Françoise Laye

\section{Françoise Laye}

\section{(2) OpenEdition}

Journals

Édition électronique

URL : http://journals.openedition.org/bulletinhispanique/2743

DOI : $10.4000 /$ bulletinhispanique.2743

ISSN : 1775-3821

Éditeur

Presses universitaires de Bordeaux

Édition imprimée

Date de publication : 28 décembre 2013

Pagination : 565-569

ISBN : 978-2-86781-908-7

ISSN : 0007-4640

Référence électronique

Françoise Laye, "Le livre de l'Intranquillité de Fernando Pessoa », Bulletin hispanique [En ligne],

115-2 | 2013, mis en ligne le 14 février 2017, consulté le 19 avril 2019. URL : http://

journals.openedition.org/bulletinhispanique/2743; DOI : 10.4000/bulletinhispanique.2743 


\title{
Le livre de l'Intranquillité de Fernando Pessoa, traduit en français par Françoise Laye ${ }^{1}$
}

\author{
FRANÇOISE LAYE \\ Traductrice
}

Nous présentons brièvement ici un ouvrage considéré comme l'œuvre maîtresse du grand poète portugais Fernando Pessoa (1888-1935), Le livre de l'Intranquillité (O Livro do Desassossego), que nous avons traduit en 1988 (1 $1^{\text {er }}$ volume) et en 1992 (2 ${ }^{\circ}$ volume). Nous mettons en relief les problèmes spécifiques de traduction que nous avons rencontrés, et certaines des solutions que nous avons tenté de leur apporter.

Mots-clés : Portugal, Pessoa, Le Livre de l'Intranquillité (O Livro do Desassossego), traduction.

Aqui tienen un breve estudio acerca del libro, quizás el más importante, del gran poeta portugués Fernando Pessoa (1888-1935), Le livre de l'Intranquillité (O Livro do Desassossego), que tradujimos en 1988 (1 $1^{\text {er }}$ volumen) y en 1992 ( $2^{\circ}$ volumen). Recalcamos algunos problemas más relevantes que encontramos en el curso de esta traducción, así como algunas de las soluciones que intentamos adoptar.

Palabras claves: Portugal, Pessoa, Le Livre de l'Intranquillité (O Livro do Desassossego), traducción.

Here is a brief study about a book considered as the most important book of the great Portuguese poet Fernando Pessoa (1888-1935), "The Book of Disquiet" (O Livro do Desassossego) that we translated in 1988 ( $1^{\text {st }}$ volume) and in $1992\left(2^{\circ}\right.$ volume). We insist upon the specific problems that appeared in the course of this translation, and some of the solutions we tried to give them.

Keywords: Portugal, Pessoa, Le Livre de l'Intranquillité (O Livro do Desassossego), translation.

1. Cette traduction a reçu le prix Eça de Queiroz de la meilleure traduction du portugais (en 1989), en même temps que l'œuvre elle-même recevait le prix du Meilleur livre étranger (en 1991).

Bulletin Hispanique, Tome 115, nº 2 - décembre 2013 - p. 565-569. 


\section{Note PRÉLIMINAIRE}

L'auteur de cette communication prie la direction et les lecteurs du Bulletin Hispanique d'excuser le caractère un peu particulier de ce texte, qui n'obéit pas rigoureusement aux normes habituelles de cette publication. En effet, ignorant que cette communication serait publiée par la suite, l'auteur a pratiquement improvisé sa contribution lors de ce congrès sur la traduction ; elle s'est, à cette occasion, partiellement inspirée d'une conférence prononcée en portugais à Braga en 2005, au cours d'un séminaire consacré aux traductions du Livro do Desassossego en diverses langues ${ }^{2}$. Le thème était donc presque identique dans les deux cas, mais l'auteur a voulu le traiter ici de façon plus large, en insistant aussi bien sur les aspects techniques et stylistiques de l'œuvre que sur l'expérience vécue de façon intense, dans la souffrance et l'angoisse, par Pessoa lui-même.

\section{BREF APERÇU HISTORIQUE}

Il convient de rappeler tout d'abord la genèse, quelque peu tortueuse, du Livro do Desassossego, dans ses diverse éditions portugaises et, par voie de conséquence, dans ses diverses traductions. Fernando Pessoa était encore pratiquement inconnu en France, jusque dans les années 1980, mises à part quelques traductions, d'ailleurs remarquables, d'Armand Guibert, parues antérieurement. Mais l'éditeur parisien Christian Bourgois décida en 1988 de publier simultanément quatre volumes de Pessoa : trois volumes de poèmes (des « hétéronymes " Reis, Caeiro et Campos), le quatrième étant Le Livre de l'Intranquillité, en prose, signé Bernardo Soares. L'édition originale était parue en 1982 chez Ática, produisant une véritable révolution dans la connaissance que l'on avait alors de l'œuvre de Pessoa. Notre traduction s'était naturellement fondée sur cette édition, mais nous avons pu, par la suite, utiliser l'excellente édition réalisée par Richard Zenith, parue en 1999 chez Assírio e Alvim, et comportant de nombreux ajouts et corrections. La deuxième édition française est parue en 1999, la troisième en 2011, toutes deux bénéficiant des nouvelles et innombrables corrections apportées au texte initial par Richard Zenith (auteur lui-même de l'une des traductions anglaises). Le succès de cette œuvre en France fut immédiat, et se poursuit aujourd'hui encore auprès d'un large public.

2. J'ai analysé certains thèmes de l'œuvre de Pessoa dans un texte publié dans un volume collectif, intitulé Pessoa l'intranquille, et comportant des études d'Eduardo Lourenço, Patrick Quillier et Richard Zenith (Christian Bourgois éditeur, 2011). Voir aussi, pour la genèse souvent dramatique de la personnalité et de l'œuvre de Pessoa, le petit volume Un singulier regard, recueil de textes autobiographiques paru aux même éditions en 2005. Enfin, une première ébauche de la présente communication a été publiée en 2006, à Braga, dans la revue Diacritica, 20-3, p. 4349, Universidade do Minho. 
Un premier problème se posa alors, soulevé par les spécialistes portugais (et quelquefois français) de Pessoa, qui critiquèrent vivement le titre de l'édition française. On soutenait que le terme "inquiétude " eût mieux convenu, en raison de sa similitude avec le portugais «inquietação ». Or, le mot " inquiétude » traduit normalement un souci concret, pour une cause précise, et ne correspond nullement au mot "desassossego ", ni à la notion d'angoisse, absolument centrale dans toute l'œuvre de Pessoa. Notons que Richard Zenith, conscient de la difficulté, a hésité, dans ses deux traductions successives, entre les termes anglais "disquiet " et "disquietude ". En ce qui me concerne, j'ai préféré créer le néologisme "intranquillité » (bien que certains en aient ensuite revendiqué la paternité!) pour tenter de rendre et de condenser divers aspects de ce texte, ondoyant autant que douloureux. Ce terme est d'ailleurs aujourd'hui totalement intégré à la langue française, tout comme son dérivé « intranquille ».

\section{LES PROBLÈMES DE TRADUCTION}

Une difficulté préalable s'est présentée, tout d'abord aux lecteurs portugais, bien entendu, puis aux divers traducteurs travaillant sur la première édition d'Ática : l'état du manuscrit, pratiquement illisible en raison de l'écriture de Pessoa, presque indéchiffrable. Il en est résulté une édition princeps parsemée d'erreurs, peut-être inévitables, mais qui rendaient bien souvent le texte totalement incompréhensible. Par la suite, comme je l'ai indiqué plus haut, les spécialistes ont progressivement corrigé les erreurs de lecture (que l'on peut chiffrer aux alentours de 800 ou 900), au fil des éditions portugaise successives, dont les diverses traductions ont pu évidemment profiter.

Mais les vrais problèmes posés par $O$ Livro do Desassossego proviennent naturellement du texte de Pessoa, d'une difficulté toute particulière en raison, d'une part, de la pensée même de l'auteur, et d'autre part de son style, que l'on peut sans exagération qualifier d'acrobatique.

Comme toute ouvre marquante, Le Livre de l'Intranquillité pose le double problème de sa thématique et de sa mise en œuvre formelle. En effet, nous abordons ici une réflexion d'une complexité et d'une subtilité rares : nous devons en découvrir les couches successives, en partant par exemple d'un superbe coucher de soleil à Lisbonne, pour nous envoler vers des couchants imaginaires en plein Pacifique, de là évoquer les puissances du rêve (thème obsessionnel chez Pessoa) pour conclure que la vie normale est totalement vaine, et beaucoup moins réelle que les mondes intérieurs que Pessoa parcourt d'un pas superbe, ici même, tout en foulant les pavés de Lisbonne. Tout cela en une seule phrase, enrichie de digressions, d'hypothèses, de contradictions, qui font ressembler la phrase - et la pensée - de Pessoa à un serpent ondulant en avant et en arrière, dérivant à droite ou à gauche, mais nous conduisant d'un mouvement sûr à une conclusion aussi brillante qu'inattendue. Il a fort bien dit : "Je n'ai jamais cru que le chemin le plus court entre A et B soit une ligne droite". 
On voit ici la difficulté pour le traducteur qui doit accompagner ce perpétuel ondoiement de la phrase, ce flot ininterrompu d'idées et d'images, tout en maintenant la souplesse, la fluidité d'un texte dont il ne doit jamais perdre l'éblouissante richesse. Mais cette langue si difficile à maîtriser n'est jamais gratuite ; elle n'est là que pour traduire la complexité d'une pensée qui se cherche et qui se présente à son tour, pourrait-on dire, en " couches de sens " successives, telles des strates géologiques partant du quotidien le plus banal pour aboutir à une réflexion de pure métaphysique.

La langue même dont use Pessoa sert admirablement ce déploiement de sens par sa splendeur, sa musicalité, la richesse de ses métaphores... et le parcours labyrinthique qu'elle impose au lecteur. Ce texte éminemment poétique présente en effet des particularités redoutables, auxquelles se sont sans doute heurtés tous les traducteurs, quoique diversement selon les " langues d'arrivée ", notamment latines ou anglo-saxonnes. La phrase de Pessoa semble tout d'abord obéir à une géométrie, à une structure rigoureuses, bientôt ruinées de l'intérieur par le foisonnement d'une pensée au parcours difficile à suivre, et les audaces d'une langue que l'auteur pousse à ses extrêmes limites : syntaxe toute personnelle, inversions dans l'ordre des mots, néologismes savoureux, tournures acrobatiques enroulant et déroulant d'immenses périodes, en vagues successives d'une poésie intense, où l'image et le rythme sont rois, et où se marient intimement la richesse des métaphores et la profondeur, l'originalité des idées que ces images semblent habiller étroitement.

\section{LA TRANSPOSITION EN FRANÇAIS}

Cette tâche se révèle particulièrement ardue du fait que notre langue se veut résolument cartésienne, rigoureusement rationnelle, en un mot cérébrale au plus haut point. Une telle langue devient un véritable carcan pour traduire un auteur tel que Pessoa : l'avalanche de métaphores stupéfiantes qui parcourent le texte et qui nous paraissent, dans la langue originale, expressives au plus haut point et parfaitement naturelles, deviennent, traduites littéralement, étranges, bizarres, presque gauches. Évoquant un moment d'insomnie, Soares dit par exemple : "durmo e desdurmo ", créant pour le second verbe un néologisme très parlant pour rendre la symétrie du passage de l'état de veille au sommeil et du sommeil à l'état de veille, avec, par la répétition des sons, la lassitude qui accompagne ce va-et-vient constant et désespérant ; " je dors et dédors " ne m'ayant pas paru acceptable euphoniquement, j'ai finalement tourné ma phrase de façon à employer les infinitifs dormir et "dédormir ", qui m’ont paru plus à même de rendre l'effet recherché. Il en va de même des phrases labyrinthiques de Pessoa, totalement opposées au " discours " naturel du français et à sa logique rectiligne. La principale difficulté était donc de transcrire en français la puissance poétique du style de Pessoa, en même temps que la rigueur bien personnelle de sa pensée, au cours souvent tortueux mais d'une logique imparable. Il s'avère donc indispensable, non pas d'adapter le texte, 
ce qui serait le défigurer, mais de le repenser en profondeur, de le transposer en usant à notre tour des ressources de notre langue pour marier la puissance poétique et l'originalité d'une pensée qui semble, à chaque phrase, se chercher et accoucher d'elle-même.

Il faut ainsi déployer à notre tour un arsenal de solutions acrobatiques, mais conforme au génie de notre langue pour reproduire celles de Pessoa, tout en leur conservant leur saveur et leur formidable originalité, leur liberté vertigineuse et leur créativité incessante.

\section{L'univers de Pessoa}

On ne peut en effet parler de la traduction des textes de Pessoa sans évoquer le monde particulier qui constitue son génie propre. Il s'agit ici, plus encore que chez les autres hétéronymes, d'un monde dominé par l'angoisse - l'angoisse de vivre, un mal-être intolérable qui ronge jusqu'en ses racines un être fragile, à qui le fait même d'exister, en soi, est insupportable ; la conscience de soi comme un supplice perpétuel, pour lequel la seule issue est le rêve, le narcissisme à outrance et ses périls, son intime perdition. Mais aussi le salut par l'art, la beauté créée par l'écriture, cette ultime salvation hautement proclamée. C'est tout cet univers contradictoire, exprimé dans une langue éblouissante, que j'ai tenté de traduire par ce terme d'intranquillité, volontairement en retrait, comme d'ailleurs le portugais desassossego, par rapport aux tourments et au désespoir exprimé tout au long d'une œuvre fascinante où, plus que jamais, comme l'endroit et l'envers d'une même étoffe, la « forme » et le " fond » ne font qu'un. 